\title{
Fiber detection for state surfaces
}

\author{
DAVID FUTER
}

\begin{abstract}
Every Kauffman state $\sigma$ of a link diagram $D(K)$ naturally defines a state surface $S_{\sigma}$ whose boundary is $K$. For a homogeneous state $\sigma$, we show that $K$ is a fibered link with fiber surface $S_{\sigma}$ if and only if an associated graph $\mathbb{G}_{\sigma}^{\prime}$ is a tree. As a corollary, it follows that for an adequate knot or link, the second and next-to-last coefficients of the Jones polynomial are the obstructions to certain state surfaces being fibers for $K$. This provides a dramatically simpler proof of a theorem of the author with Kalfagianni and Purcell.
\end{abstract}

57M25, 57M27, 57M50

\section{Introduction}

In the 1930s, Seifert [15] gave an algorithm that starts with a link diagram $D(K)$ and produces an orientable surface whose boundary is $K$. The algorithm works as follows. For every crossing of $D$, smooth the diagram near the crossing by following an orientation on $K$. This gives a disjoint union of circles in the projection plane. These circles bound a number of disks, disjointly embedded in the ball below the projection plane. Then, these disks can be jointed by half-twisted bands at the crossings to give a surface $S \subset S^{3}$, such that $\partial S=K$.

Seifert's construction has a natural generalization. At each crossing, there are two possible smoothings, or resolutions of the crossing, as depicted in Figure 1. A Kauffman state is a choice of $A$-or $B$-resolution at each crossing. As in Seifert's construction, a state $\sigma$ gives rise to a union of circles in the projection plane. These circles bound disjoint disks, which can be joined by half-twisted bands to give a state surface $S_{\sigma}$. See Figure 2.

Another common example of a state surface is the two checkerboard surfaces of a diagram. The regions in the complement of $D(K)$ can be checkerboard colored, black and white. If the state $\sigma$ traces the boundaries of all the black regions, then joining these regions together produces the black checkerboard surface. Making the opposite choice for $\sigma$ produces the white surface. These checkerboard surfaces have been studied since the time of Tait in the 19th century; see Przytycki [14] for a survey. In the special case 


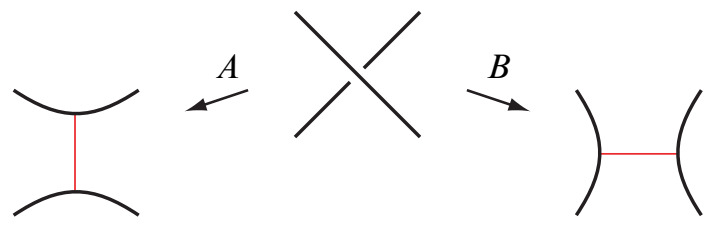

Figure 1: $A$ - and $B$-resolutions at a crossing of $D$

of alternating diagrams, the checkerboard surfaces correspond to choosing the all- $A$ or all- $B$ state for $\sigma$.

The goal of this paper is to characterize when a state surface $S_{\sigma}$ is a fiber in a fibration of $S^{3} \backslash K$ over $S^{1}$. For alternating diagrams, the strikingly simple answer is that the all- $A$ surface $S_{A}$ is a fiber if and only if $D$ is a connected sum of positive 2-braids. (See Lemma 4 below.) Stating our result in general requires a handful of definitions.

If every crossing of $D(K)$ is resolved as in Figure 1, according to a state $\sigma$, then the crossing point in the projection plane lies in a region complementary to the state circles of $\sigma$. These state circles partition the projection plane into regions. The state $\sigma$ is called homogeneous if all crossings in the same region carry the same ( $A$ or $B$ ) resolution. For example, the state shown in Figure 2 is homogeneous. This notion was introduced by Cromwell [2] for the Seifert state, and easily extends to other states.
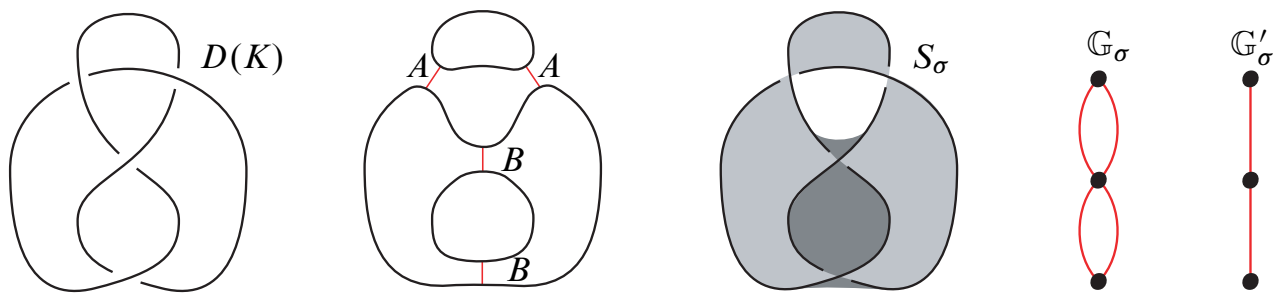

Figure 2: Left to right: A diagram $D(K)$. A homogeneous state $\sigma$, coming from Seifert's algorithm. The state surface $S_{\sigma}$ corresponding to $\sigma$. The graph $\mathbb{G}_{\sigma}$ embeds into $S_{\sigma}$ as a spine. The reduced graph $\mathbb{G}_{\sigma}^{\prime}$.

The choices that lead to a Kauffman state $\sigma$ can be conveniently encoded in a state graph $\mathbb{G}_{\sigma}$. This graph has one vertex for each state circle of $\sigma$. Each crossing $x$ of $D$ gives rise to an edge between the state circles at the resolution of $x$. (In Figures 1 and 2, these edges are shown in red, lighter than the link projection.) From the graph $\mathbb{G}_{\sigma}$, we construct a reduced graph $\mathbb{G}_{\sigma}^{\prime}$ by removing all duplicate edges between a pair of vertices. Our main result is that this reduced graph carries fibering information. 
Theorem 1 Let $\sigma$ be a homogeneous state of a link diagram $D(K)$. Then $S^{3} \backslash K$ fibers over the circle with fiber $S_{\sigma}$ if and only if the reduced graph $\mathbb{G}_{\sigma}^{\prime}$ is a tree.

The topology of state surfaces was recently studied by Ozawa [13]. For homogeneous states, he showed that the surface $S_{\sigma}$ is essential in $S^{3} \backslash K$ if and only the state $\sigma$ is adequate, meaning that $\mathbb{G}_{\sigma}$ has no 1 -edge loops (equivalently, $\mathbb{G}_{\sigma}^{\prime}$ has no 1-edge loops). Since a tree has no loops of any length, all the states where $S_{\sigma}$ is a fiber must be adequate.

In the special case where $\sigma$ is the all- $A$ or all- $B$ state, the graphs $\mathbb{G}_{A}^{\prime}$ and $\mathbb{G}_{B}^{\prime}$ are particularly worthy of attention due to their connection to the Jones polynomial of $K$. This is a Laurent polynomial invariant of $K$, which can be written in the form

$$
J_{K}(t)=\alpha t^{k}+\beta t^{k-1}+\cdots+\beta^{\prime} t^{m+1}+\alpha^{\prime} t^{m},
$$

so that the second and next-to-last coefficients of $J_{K}(t)$ are $\beta$ and $\beta^{\prime}$, respectively. Stoimenow [17] and Dasbach and Lin [3] have observed that if $D$ is $A$-adequate (meaning the all- $A$ state is adequate), then $\left|\beta^{\prime}\right|=1-\chi\left(\mathbb{G}_{A}^{\prime}\right)$. Similarly, if $D$ is $B$-adequate, then $|\beta|=1-\chi\left(\mathbb{G}_{B}^{\prime}\right)$. As a result, the following corollary immediately follows from Theorem 1 .

Corollary 2 Let $K$ be a link that admits a connected, $A$-adequate diagram $D$. Then $S^{3} \backslash K$ fibers over $S^{1}$ with fiber the state surface $S_{A}=S_{A}(D)$ if and only if $\beta^{\prime}=0$.

In other words, the next-to-last Jones coefficient $\beta^{\prime}$ is precisely the obstruction to $S^{3} \backslash K$ being fibered with fiber surface $S_{A}$. Similarly, for $B$-adequate links, the second coefficient $\beta$ is the obstruction to $S^{3} \backslash K$ being fibered with fiber surface $S_{B}$.

Ever since Jones introduced his knot polynomial in the 1980s, it has been a tantalizing open problem to understand exactly what this invariant and its generalizations say about the topology of knot and link complements. The first rigorous relations of this sort have appeared in the last few years, in the work of Dasbach and Lin [4], Garoufalidis [9] and Futer, Kalfagianni and Purcell $[5 ; 6 ; 7]$. Corollary 2 is one of the more striking connections between quantum knot invariants and classical geometric topology to have been found so far.

Theorem 1 and Corollary 2 were first proved in the author's joint work with Kalfagianni and Purcell [7, Theorem 5.21 and Corollary 9.16]. However, the proof of [7, Theorem 5.21] appears over 80 pages into the monograph. That proof relies on the detailed study of a polyhedral decomposition of $S^{3} \backslash S_{\sigma}$, and much effort is expended to show that the polyhedra have desirable properties [7, Chapters 2-4]. This polyhedral 
decomposition is also used to gain a detailed understanding of $I$-bundles in $S^{3} \backslash S_{\sigma}$ and the hyperbolic geometry of $S^{3} \backslash K$.

By contrast, the proof of Theorem 1 contained in this paper is short and direct. As a consequence, one also obtains a short and readily digestible proof of Corollary 2.

Our proof builds up the surface $S_{\sigma}$ inductively via Murasugi sums (see Figure 5), applying results of Gabai [8] to deduce fibering information. This inductive approach is very similar in spirit to the methods used by Ozawa [13]. It is also fruitfully exploited in a recent paper of Girão [10] to prove a fibering criterion for augmented links.

\section{Proof}

Before beginning the main proof of Theorem 1, we wish to dismiss a few special cases. If $D(K)$ depicts an unknot with no crossings, then $\mathbb{G}_{\sigma}^{\prime}$ is a single vertex, and the spanning disk $S_{\sigma}$ is a fiber of the solid torus $S^{3} \backslash K$. Thus the theorem holds trivially. If $D(K)$ is a split diagram, then $\mathbb{G}_{\sigma}^{\prime}$ is a disconnected graph (which cannot be a tree), and $S^{3} \backslash K$ is reducible (hence cannot be fibered). Again, the theorem holds trivially in this case.

If the state $\sigma$ is not adequate, ie $\mathbb{G}_{\sigma}$ has a 1-edge loop, the reduced graph $\mathbb{G}_{\sigma}^{\prime}$ cannot be a tree. In this case, the surface $S_{\sigma}$ is nonorientable, hence cannot be a fiber. Thus the theorem holds for nonadequate states.

For the remainder of the paper, we work under the assumptions that $D(K)$ is connected and has at least one crossing, and that the state $\sigma$ is adequate. With these simplifying assumptions, the proof of Theorem 1 proceeds by induction on the number of cut vertices in the graph $\mathbb{G}_{\sigma}^{\prime}$. Recall that a cut vertex is a vertex that separates $\mathbb{G}_{\sigma}^{\prime}$.

The base case of the induction involves prime, alternating diagrams. Recall that a link diagram $D(K)$ is called prime if, for every simple closed curve $\gamma$ in the projection plane that intersects $D(K)$ transversely in two points, one of the two sides of $\gamma$ contains no crossings. In other words, $D(K)$ fails to be prime precisely when it is the connected sum of two nontrivial diagrams.

Lemma 3 Let $\sigma$ be a homogeneous state of $D(K)$. Then $\mathbb{G}_{\sigma}^{\prime}$ does not contain any cut vertices if and only if all of the following hold: $D(K)$ is prime and alternating, and $\sigma$ is the all- $A$ or all- $B$ state.

Proof For the "if" direction, suppose that $D(K)$ is prime and alternating, and $\sigma$ is the all- $A$ or all- $B$ state. Without loss of generality, $\sigma$ is the all- $A$ state. Then $\mathbb{G}_{\sigma}$ is 


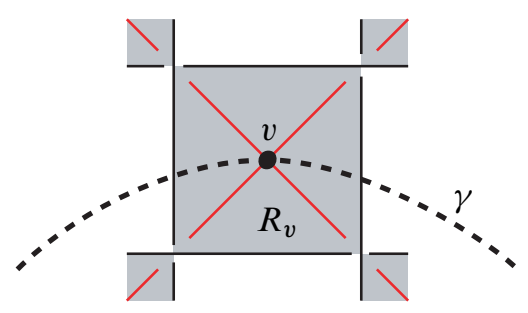

Figure 3: If $D(K)$ is an alternating diagram, and $v$ is a cut vertex of the checkerboard graph $\mathbb{G}_{A}$, then the corresponding black region $R_{v}$ separates the diagram as a connected sum (with summands on opposite sides of $\gamma$ )

the all- $A$ checkerboard graph of $D$. The graph $\mathbb{G}_{\sigma}=\mathbb{G}_{A}$ naturally embeds as a spine of the (black) checkerboard surface $S_{\sigma}=S_{A}$, with one vertex in each black region of $D(K)$ and one edge running through each half-twisted band.

Suppose, for a contradiction, that $v \in \mathbb{G}_{A}^{\prime}$ is a cut vertex. Then $v$ also separates the unreduced graph $\mathbb{G}_{A}$. Since $\mathbb{G}_{A}$ is embedded as a spine of the checkerboard surface $S_{A}$, the black region $R_{v}$ corresponding to $v$ must separate the checkerboard surface, hence also separate the diagram $D(K)$. In other words, there is a simple closed curve $\gamma$ in the projection plane such that the only black region met by $\gamma$ is $R_{v}$, and such that each component of $\mathbb{R}^{2} \backslash \gamma$ contains at least one crossing (these crossings correspond to edges of $\left.\mathbb{G}_{A} \backslash v\right)$. This curve decomposes $D(K)$ as a connected sum, violating the hypothesis of primeness. See Figure 3.
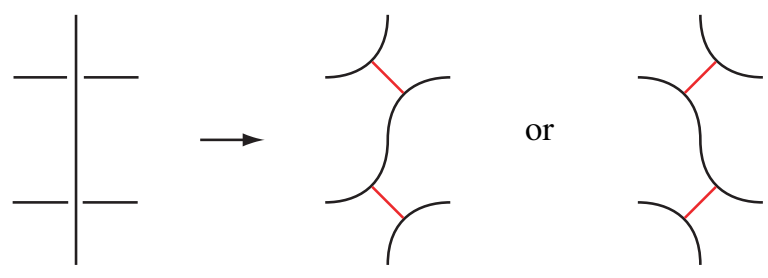

Figure 4: If $D(K)$ is a nonalternating diagram, a homogeneous resolution of an over-over strand of $D$ must result in a state circle that has other circles on both sides. The same is true of an under-under strand.

For the "only if" direction, suppose that $D(K)$ is not alternating. Since $\sigma$ is homogeneous, some state circle of $\sigma$ (corresponding to a nonalternating segment of $D$ ) must have other state circles on both sides. See Figure 4 . The corresponding vertex of $\mathbb{G}_{\sigma}$ will separate $\mathbb{G}_{\sigma}$, and also $\mathbb{G}_{\sigma}^{\prime}$. Thus, if $\mathbb{G}_{\sigma}^{\prime}$ contains no cut vertices, $D(K)$ must be alternating. 
If $D$ is alternating but $\sigma$ is not the all- $A$ or all- $B$ state, then some state circle fails to follow the boundary of a region of $D$. Just as in Figure 4, this implies that this state circle has other circles on both sides, hence $\mathbb{G}_{\sigma}^{\prime}$ has a cut vertex.

Finally, if $D$ is not prime, then there is a simple closed curve $\gamma$ that meets only one white region and only one black region, with crossings on both sides of $\gamma$. One of these regions corresponds to a vertex of $\mathbb{G}_{\sigma}$ (depending on whether $\sigma$ is the all- $A$ or all- $B$ state), and this vertex separates $\mathbb{G}_{\sigma}^{\prime}$. Therefore, if $\mathbb{G}_{\sigma}^{\prime}$ has no cut vertices, $D(K)$ must be prime and alternating, with $\sigma$ the all- $A$ or all- $B$ state.

The base case of the induction is the following lemma.

Lemma 4 Suppose $D(K)$ is a prime, alternating diagram with at least one crossing. Then the following are equivalent for the all- $A$ state:

(1) $\mathbb{G}_{A}^{\prime}$ is a tree.

(2) $\mathbb{G}_{A}$ has exactly two vertices.

(3) $D(K)$ is a positive 2-braid.

(4) The checkerboard surface $S_{A}$ is a fiber in $S^{3} \backslash K$.

The same equivalence holds for $S_{B}, \mathbb{G}_{B}^{\prime}$ and negative 2-braids.

Proof Suppose $\mathbb{G}_{A}^{\prime}$ is a tree. If it has only one vertex, then there are no edges, contradicting the hypothesis that $D(K)$ has crossings. If it has three or more vertices, some vertex will be separating, contradicting Lemma 3 . Thus $\mathbb{G}_{A}$ has exactly two vertices, giving (1) $\Rightarrow(2)$.

If $\mathbb{G}_{A}$ has two vertices, then $D(K)$ has exactly two black regions, connected to each other at each crossing. This is the diagram of a positive 2-braid. Conversely, if $D(K)$ is a positive 2-braid, then $\mathbb{G}_{A}^{\prime}$ is a stick with two vertices, which is a tree. Thus (1) $\Leftrightarrow(2) \Leftrightarrow(3)$.

For $(3) \Rightarrow(4)$, assume that $D(K)$ is a positive 2 -braid. Then $K$ is a $(2, n)$ torus knot or link, and it is well-known that $S_{A}$ is a fiber (Stallings [16]).

For $(4) \Rightarrow(3)$, recall that if $D$ is alternating, prime, and not a 2-braid, then $S^{3} \backslash K$ admits a hyperbolic structure (Menasco [12]). Then, Adams [1, Theorem 1.9] shows that the checkerboard surface $S_{A}$ is not a fiber. 
Remark 5 There is also a more direct proof that $(3) \Leftrightarrow(4)$, which does not rely on hyperbolic geometry. Instead, this alternate argument relies on Menasco's decomposition of $S^{3} \backslash K$ into two ideal polyhedra [11]. The 1-skeleton of each polyhedron is isomorphic (as a planar graph) to the projection graph of $D(K)$. In particular, the faces of the polyhedra can be 2-colored: the union of all the black faces is the checkerboard surface $S_{A}$, while the union of all the white faces is the checkerboard surface $S_{B}$. In particular, the manifold $S^{3} \backslash S_{A}$ can be obtained by gluing the two polyhedra along white faces only.

For (3) $\Rightarrow(4)$, suppose $D$ is a positive 2-braid. Then every white face is a bigon. In other words, every polyhedron is combinatorially a prism $P \times I$, where $P$ is an ideal polygon, and the lateral faces are the white bigons. The product structure of $P \times I$ extends as we glue the two polyhedra along their lateral faces, implying that $S^{3} \backslash S_{A} \cong S_{A} \times I$, hence $S_{A}$ is a fiber.

For (4) $\Rightarrow$ (3), suppose $S^{3} \backslash S_{A} \cong S_{A} \times I$. Then, [7, Lemma 4.17] shows that Menasco's polyhedral decomposition must "see" this product structure: every white face must be a product of an ideal edge with $I$, ie an ideal bigon. Note that when the proof of that lemma is applied to Menasco's well-known polyhedral decomposition, it becomes self-contained, and does not require the machinery developed in [7]. If every white $B$-region of $D(K)$ is a bigon, these bigons must be joined end to end, implying that $D(K)$ is a positive 2 -braid.

We are now ready to complete the proof of the main theorem.
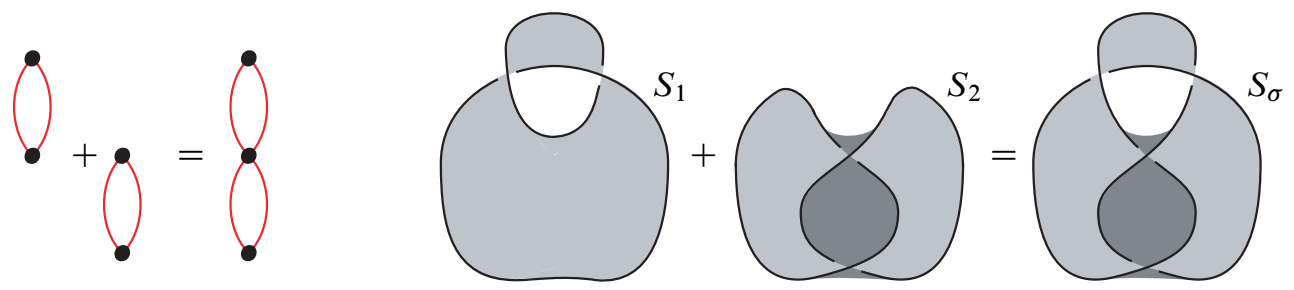

Figure 5: Left: The graph $\mathbb{G}_{\sigma}$ decomposes as a union of subgraphs $\mathbb{G}_{1}$ and $\mathbb{G}_{2}$, joined along the cut vertex $v$. Right: The corresponding decomposition of $S_{\sigma}$ as a Murasugi sum of state surfaces $S_{1}$ and $S_{2}$.

Proof of Theorem 1 We proceed by induction on $n$, where $n$ is the number of cut vertices in $\mathbb{G}_{\sigma}^{\prime}$. For the base case, let $n=0$, and recall the running assumption that $D(K)$ has at least one crossing. Then Lemma 3 says that the diagram $D(K)$ is prime and alternating, and $\sigma$ is the all- $A$ or all- $B$ state. By Lemma $4, S_{\sigma}$ is a fiber if and only if $\mathbb{G}_{\sigma}^{\prime}$ is a tree, as desired. 
For the inductive step, suppose $n>0$ and $v$ is a cut vertex of $\mathbb{G}_{\sigma}^{\prime}$. Then $\mathbb{G}_{\sigma}^{\prime}=\mathbb{G}_{1}^{\prime} \cup_{v} \mathbb{G}_{2}^{\prime}$, where $\mathbb{G}_{1}^{\prime}$ and $\mathbb{G}_{2}^{\prime}$ are subgraphs that are disjoint except at $v$. The unreduced graph $\mathbb{G}_{\sigma}$, which has the same adjacency relations as $\mathbb{G}_{\sigma}^{\prime}$, also decomposes as a union of subgraphs $\mathbb{G}_{1}$ and $\mathbb{G}_{2}$ that are disjoint except at $v$. See Figure 5, left. On the diagrammatic side, $D(K)$ decomposes as the Murasugi sum of diagrams $D\left(K_{1}\right)$ and $D\left(K_{2}\right)$, and the state surface $S_{\sigma}$ decomposes as the Murasugi sum of state surfaces $S_{1}$ and $S_{2}$. See Figure 5, right.

Note that the Kauffman state $\sigma_{i}$ that gives rise to $S_{i}$ is obtained by restricting $\sigma$ to the crossings of $D\left(K_{i}\right)$. Thus each $S_{i}$ is the state surface of a homogeneous state, with reduced graph $\mathbb{G}_{i}^{\prime}$. Note as well that each $\mathbb{G}_{i}^{\prime}$ has fewer than $n$ cut vertices. Thus, by the inductive hypothesis, $S_{i}$ is a fiber in $S^{3} \backslash K_{i}$ if and only if $\mathbb{G}_{i}^{\prime}$ is a tree.

Now, we recall Gabai's Theorem [8]: $S_{\sigma}$ is a fiber if and only if each $S_{i}$ is a fiber. Clearly, $\mathbb{G}_{\sigma}^{\prime}$ is a tree if and only if each $\mathbb{G}_{i}^{\prime}$ is a tree. This completes the proof.

\section{Acknowledgements}

The author thanks the NSF for its support via grant number DMS-1007221.

\section{References}

[1] C Adams, Noncompact Fuchsian and quasi-Fuchsian surfaces in hyperbolic 3manifolds, Algebr. Geom. Topol. 7 (2007) 565-582 MR2308957

[2] P R Cromwell, Homogeneous links, J. London Math. Soc. 39 (1989) 535-552 MR1002465

[3] O T Dasbach, X-S Lin, On the head and the tail of the colored Jones polynomial, Compos. Math. 142 (2006) 1332-1342 MR2264669

[4] O T Dasbach, X-S Lin, A volumish theorem for the Jones polynomial of alternating knots, Pacific J. Math. 231 (2007) 279-291 MR2346497

[5] D Futer, E Kalfagianni, J S Purcell, Dehn filling, volume, and the Jones polynomial, J. Differential Geom. 78 (2008) 429-464 MR2396249

[6] D Futer, E Kalfagianni, J S Purcell, Slopes and colored Jones polynomials of adequate knots, Proc. Amer. Math. Soc. 139 (2011) 1889-1896 MR2763776

[7] D Futer, E Kalfagianni, J Purcell, Guts of surfaces and the colored Jones polynomial, Lecture Notes in Mathematics 2069, Springer, Heidelberg (2013) MR3024600

[8] D Gabai, The Murasugi sum is a natural geometric operation, from: "Low-dimensional topology", (S J Lomonaco, Jr, editor), Contemp. Math. 20, Amer. Math. Soc. (1983) 131-143 MR718138 
[9] S Garoufalidis, The Jones slopes of a knot, Quantum Topol. 2 (2011) 43-69 MR2763086

[10] D Girão, On the fibration of augmented link complements, to appear in Geom. Dedicata

[11] W W Menasco, Polyhedra representation of link complements, from: "Low-dimensional topology", (S J Lomonaco, Jr, editor), Contemp. Math. 20, Amer. Math. Soc. (1983) 305-325 MR718149

[12] W Menasco, Closed incompressible surfaces in alternating knot and link complements, Topology 23 (1984) 37-44 MR721450

[13] M Ozawa, Essential state surfaces for knots and links, J. Aust. Math. Soc. 91 (2011) 391-404 MR2900614

[14] J H Przytycki, From Goeritz matrices to quasi-alternating links, from: "The mathematics of knots", (M Banagl, D Vogel, editors), Contrib. Math. Comput. Sci. 1, Springer, Heidelberg (2011) 257-316 MR2777853

[15] H Seifert, Über das Geschlecht von Knoten, Math. Ann. 110 (1935) 571-592 MR1512955

[16] J R Stallings, Constructions of fibred knots and links, from: "Algebraic and geometric topology, Part 2", (R J Milgram, editor), Proc. Sympos. Pure Math. 32, Amer. Math. Soc. (1978) 55-60 MR520522

[17] A Stoimenow, Coefficients and non-triviality of the Jones polynomial, J. Reine Angew. Math. 657 (2011) 1-55 MR2824782

Department of Mathematics, Temple University

Philadelphia, PA 19122, USA

dfuter@temple.edu

http://math.temple.edu/ dfuter

Received: 3 May 2012 Revised: 27 March 2013 